PROCEEDINGS OF THE

AMERICAN MATHEMATICAL SOCIETY

Volume 136, Number 8, August 2008, Pages 2933-2936

S 0002-9939(08)09329-5

Article electronically published on March 14, 2008

\title{
RESTRICTIONS OF THE DISC ALGEBRA DESCRIBED LOCALLY
}

\author{
ARNE STRAY \\ (Communicated by Juha M. Heinonen)
}

\section{INTRODUCTION}

Let $D$ denote the open unit disc in the complex plane and denote by $T$ the unit circle. We consider a closed subset $X$ of $D \cup T$ such that $X \cap T$ has zero linear measure and such that $X \cap D$ is a Blaschke sequence, meaning that $X \cap D$ is a sequence $S=\left\{z_{\nu}\right\}$ satisfying $\sum_{\nu}\left(1-\mid z_{\nu \mid}\right)<\infty$.

The aim of the present paper is to give an answer to the following question: Is there a local charazterization of the functions $f$ on $X$ admitting a continuous extension $\tilde{f}$ to $D \cup T$ with $\tilde{f}$ analytic in $D$ ?

Let $A(D)$ denote the disc algebra consisting of all continuous functions on $D \cup T$ being analytic in $D$. Our question is to describe the restriction space $\left.A\right|_{X}$ locally.

Of course if $\left.A\right|_{X}=C(X)$, the space of all continuous functions on $X$, we already have a local description since continuity is a local property. But it is a well known fact that $\left.A\right|_{X}=C(X)$ holds if and only if $S=\left\{z_{\nu}\right\}$ is an interpolating sequence for the algebra $H^{\infty}(D)$ consisting of all bounded analytic functions in $D$, meaning that the restriction map $\left.f \Rightarrow f\right|_{X \cap D}$ takes $H^{\infty}(D)$ onto the sequence space $l^{\infty}$. See for example 4, where the problem is studied in a more general context. Moreover, L. Carleson (see 2], Chapter VII, or [1, page 149) characterized the interpolating sequences by the condition

$$
i n f_{\mu} \prod_{\nu \neq \mu}\left|\frac{z_{\mu}-z_{\nu}}{1-z_{\mu} \overline{z_{\nu}}}\right|>0 .
$$

As is well known, the interpolating sequences for $H^{\infty}(D)$ form a rather exclusive subclass of the Blaschke sequences, and so for an arbitrary Blaschke sequence Carleson's condition doesn't seem to help.

Interpolation by bounded analytic functions can be studied using Carleson's condition or the more classical Nevanlinna-Pick theory (see [2, page 7 and pages 165174). The latter gives conditions on when a bounded function $h$ on $X \cap D$ extends to a function $\tilde{h}$ in $H^{\infty}(D)$. For our problem, however, the Nevanlinna-Pick theorem has two drawbacks: it is not a local condition, and it says nothing about when the extended function belongs to $A(D)$.

Received by the editors September 13, 2006, and, in revised form, August 21, 2007.

2000 Mathematics Subject Classification. Primary 30E05.

The author was supported by the Norwegian Research Council, grant 177355 , and by the ESF Research Networking Programme HCAA.

(C)2008 American Mathematical Society Reverts to public domain 28 years from publication 
Neverthless our problem will be solved by combining the two theories: Our main result is formulated using a local Nevanlinna-Pick condition, while the proof depends heavily on Carleson's corona theorem.

\section{MAin RESUlt}

As in the introduction we write $S=\left\{z_{\nu}\right\}$. Given interpolation data $\left\{w_{\nu}\right\}$ on $S$, the data will be called smooth with respect to $\left\{H^{\infty}(D),\left\{z_{\nu}\right\}\right\}$ if the following condition holds:

For any $\epsilon>0$ and any $\zeta \in X$, there exist a constant $c_{\zeta}$ and a disc $D_{\zeta}$ centered at $\zeta$ and an analytic function $h$ in $D \cap D_{\zeta}$ such that $|h| \leq \epsilon$ and $h\left(z_{\nu}\right)=w_{\nu}-c_{\zeta}$ for $z_{\nu} \in D \cap D_{\zeta}$.

In other words, the data are smooth if they have a small local oscillation measured by a local interpolation problem of Nevanlinna-Pick type.

Theorem 1. The restriction space $\left.A(D)\right|_{X}$ consists of all $f \in C(X)$ such that the data $\left\{f\left(z_{\nu}\right)\right\}$ are smooth with respect to $\left\{H^{\infty}(D),\left\{z_{\nu}\right\}\right\}$.

Proof. Let $\left\{w_{\nu}\right\}$ denote data being smooth with respect to $H^{\infty}(D),\left\{z_{\nu}\right\}$. It is easy to see that the constant $c_{\zeta}$ in the definition of smoothness can be selected independent of $\epsilon$ and that the function $f$ taking the values $w_{\nu}$ at $z_{\nu}$ and $c_{\zeta}$ at $\zeta \in \bar{S} \cap T$ is continuous on $\bar{S}$. We may assume that $f$ is extended to be continuous on $X$.

Now we apply the Rudin Carleson theorem (see [3], page 81) and find $g \in A(D)$ such that $g=f$ on $X \cap T$. The modified data $u_{\nu}=w_{\nu}-g\left(z_{\nu}\right)$ are of course also smooth, and our theorem is proved if we can find $f_{1} \in A(D)$ such that $f_{1}\left(z_{\nu}\right)=$ $u_{\nu}, \nu=1,2, \ldots$.

We claim that given $\epsilon>0$, there is $h \in H^{\infty}(D)$ and an integer $N$ such that $h\left(z_{\nu}\right)=u_{\nu}, \nu \geq N$ and such that $\|h\|_{\infty}<\epsilon$ where $\|h\|_{\infty}=\sup \{|h(z)|$, $z \in D\}$.

To justify the claim, we note that since $\bar{S} \cap T$ has zero linear measure, there are disjoint discs $D_{1}, D_{2}, \ldots, D_{k}$ covering $\bar{S} \cap T$ such that the interpolation problem $s\left(z_{\nu}\right)=u_{\nu}$ can be solved for $z_{\nu} \in \bigcup_{j=1}^{j=k} D_{j}$, with $s$ being analytic and bounded by $\epsilon$ in $D \cap \bigcup_{j=1}^{j=k} D_{j}$. If $N$ is a sufficiently large integer, the Blaschke product $B_{N}$ corresponding to $S_{N}=\left\{z_{\nu}: \nu \geq N\right\}$ will satisfy

$$
\left\{z:\left|B_{N}(z)\right|<\frac{1}{2}\right\} \subset \bigcup_{j=1}^{j=k} D_{j} .
$$

Then the claim follows from Carleson's lemma (see Lemma 2 in [1, page 203), which we state here in a somewhat specialized form:

Lemma 1. Let $B$ denote a Blaschke product and let $W=\left\{z:|B(z)|<\frac{1}{2}\right\}$. There is an absolute constant $C$ such that if $H$ is analytic and bounded by one in modulus on $W$, there is $h \in H^{\infty}(D)$ such that $\|h\|_{\infty} \leq C$ and satisfying $h=H$ at the zeros of $B$.

Now we choose a sequence $\left\{r_{j}\right\} \subset[0,1)$, strictly increasing to 1 , with $r_{1}=0$, and define $S_{j}=S \cap\left\{z: r_{j} \leq|z|<r_{j+1}\right\}, j=1,2, \ldots$. Our aim is to find $f \in A(D)$ such that $f=0$ on $X \cap T$ and $f\left(z_{\nu}\right)=u_{\nu}$ for all $\nu$. 
We first do the interpolation on $\bigcup_{j=1}^{\infty} S_{2 j-1}$. For each $k$, let $B_{k}$ denote the Blaschke product corresponding to $S_{k}$, so that

$$
B_{k}(z)=\prod_{z_{\nu} \in S_{k}} \frac{\left|z_{\nu}\right|}{-z_{\nu}} \frac{z-z_{\nu}}{1-\overline{z_{\nu}} z}
$$

We can arrange it such that

$$
\text { (1) }:\left\{z:\left|B_{k}(z)\right|<\frac{1}{2}\right\} \cap\left\{z: \prod_{|m-k| \geq 2}\left|B_{m}(z)\right|<\frac{1}{2}\right\}=\emptyset
$$

for all $k$.

We claim that there is a function $a_{k} \in A(D)$ with the following properties:

$a_{k}=0$ on $X \cap T \bigcup\left(\bigcup_{|m-k| \geq 2} S_{m}\right), a_{k}=1$ on $S_{k}$, and $\left\|a_{k}\right\|_{\infty} \leq A$, where $A$ is an absolute constant.

The claim is justified as follows:

Since $X \cap T$ is a peak set for $A(D)$, there is $p_{k} \in A(D)$ such that $p_{k}=1$ on $X \cap T$ while $\left|p_{k}\right|<1$ on $\bar{D} \backslash(X \cap T)$. But with $n$ large enough, we get

$$
\left|\left(1-p_{k}^{n}\right) \prod_{|m-k| \geq 2}\right| B_{m}(z) \mid \geq \frac{1}{2},
$$

where $\left|B_{k}(z)\right|<\frac{1}{2}$, and Carleson's lemma gives that $\left(\left(1-p_{k}^{n}\right) \prod_{|m-k| \geq 2} B_{m}(z)\right)^{-1}$ can be interpolated on $S_{k}$ by a function in $H^{\infty}(D)$ bounded by an absolute constant. Since $S_{k}$ is finite, the minimal norm interpolating function is, by the classical Nevanlinna-Pick theorem, a complex multiple $\lambda_{k} \beta_{k}$ of a finite Blaschke product $\beta_{k}$, and the claim is thus proved with $a_{k}$ of the form

$$
a_{k}=\lambda_{k} \beta_{k}\left\{\left(1-p_{k}^{n}\right) \prod_{|m-k| \geq 2} B_{m}(z)\right\} .
$$

We may assume that the sequence $\left\{r_{j}\right\}$ is chosen such that there is $b_{k} \in A(D)$ such that $b_{k}\left(z_{\nu}\right)=u_{\nu}$ for $z_{\nu} \in S_{k}$ and $\left\|b_{k}\right\|_{\infty}<2^{-k}, k=1,2, \ldots$. This follows easily from the above claim combined with the Nevanlinna-Pick theorem.

We thus get an interpolating function

$$
F=\sum a_{2 k-1} b_{2 k-1}
$$

taking correct values on $\bigcup_{k=1}^{\infty} S_{2 k-1}$.

Let $\prod$ denote the Blaschke product corresponding to $\bigcup S_{2 k-1}$. We want to repeat our interpolation argument on $\bigcup S_{2 k}$. In order to do that, we have to show that the data $\frac{u_{\mu}-F\left(z_{\mu}\right)}{\prod\left(z_{\mu}\right)}$ are smooth with respect to $\left\{H^{\infty}(D),\left\{z_{\mu}\right\}\right\}$. (Here $z_{\mu}$ should be understood as the generic point of $\bigcup S_{2 k}$ and $u_{\mu}$ denotes the corresponding value to be interpolated.)

Given $\epsilon>0$, we choose $h_{\epsilon} \in H^{\infty}(D)$ such that $h_{\epsilon}\left(z_{\nu}\right)=u_{\nu}$ for $\nu \geq N$, where $N$, is so large that we may assume $\left\|h_{\epsilon}\right\|_{\infty}<\epsilon$. Now let $F_{N}=\sum_{N}^{\infty} a_{2 k-1} b_{2 k-1}$. Then we can write

$$
h_{\epsilon}-F_{N}=\prod_{N} G
$$

where $\prod_{N}$ is a cofinite subproduct of $\prod$ and where we may assume that $\|G\|_{\infty}<2 \epsilon$. Suppose $\prod_{N} \Pi^{N}=\prod$. Then we have

$$
\frac{h_{\epsilon}-F_{N}}{\Pi}=\frac{G}{\Pi^{N}} .
$$


From Carleson's lemma ([1], Lemma 2, page 203) it now follows easily that $\frac{G}{\Pi^{N}}$ can be interpolated at the points $z_{\mu}, \mu>M$ by a function $g \in H^{\infty}(D)$ with $\|g\|_{\infty}<A\|G\|_{\infty}<2 A \epsilon$, where $A$ is an absolute constant, provided $M$ is large enough. If

$$
\beta_{M}(z)=\prod_{\mu \geq M} \frac{\left|z_{\mu}\right|}{-z_{\mu}} \frac{z-z_{\mu}}{1-\overline{z_{\mu}} z}
$$

we only have to observe that

$$
\left\{z:\left|\beta_{M}(z)\right|<\frac{1}{2}\right\} \cap\left\{z:\left|\prod^{N}(z)\right|<\frac{1}{2}\right\}=\emptyset
$$

for $M$ large, since $\prod^{N}$ is a finite Blaschke product.

This means that the data $\frac{u_{\mu}-F\left(z_{\mu}\right)}{\prod\left(z_{\mu}\right)}$ are smooth with respect to $\left\{H^{\infty}(D),\left\{z_{\mu}\right\}\right\}$. In the same way as we found $F \in A(D)$ solving the original data on $\bigcup_{k=1}^{\infty} S_{2 k-1}$, we find $F_{1} \in A(D)$ interpolating $\left\{\frac{u_{\mu}-F\left(z_{\mu}\right)}{\Pi\left(z_{\mu}\right)}\right\}$ on $\left\{z_{\mu}\right\}$. To obtain $F_{1}$ a minor modification of the construction of $F$ is needed: We found $F=\sum a_{2 k-1} b_{2 k-1}$, where $b_{2 k-1}$ solved a finite interpolation problem on $S_{2 k-1}$ with the bound $\left\|b_{2 k-1}\right\|_{\infty}<2^{-k}$. We cannot be sure that a similar bound exists for interpolation of the new data on $\left\{S_{2 k}\right\}$, but if the local interpolation is done on finite unions of the sets $S_{2 k}$, the necessary estimates will hold. With $F_{1}$ at hand, finally $F+\prod F_{1} \in A(D)$ solves the original problem.

\section{REFERENCES}

[1] P. Duren, Theory of $H^{p}$-spaces, Academic Press, 1970. MR0268655 (42:3552)

[2] J. B. Garnett, Bounded analytic functions, Academic Press, 1981. MR628971 (83g:30037)

[3] K. Hoffman, Banach spaces of analytic functions, Prentice Hall, 1962. MR0133008 (24a:2844)

[4] M. Hasumi, Interpolation sets for logmodular Banach algebras, Osaka J. Math 3, 1966, pp. 303-311. MR0209842(35:738)

Department of Mathematics, University of Bergen, Allegt 55, N-5000 Bergen, NorWAY 